\title{
Flottement Du Taux De Change Et Incomplétude De La Transmission De La Politique Monétaire Au Taux Débiteur
}

\author{
Hassan Hachimi Alaoui \\ Enseignant chercheur - Université Ibn Zohr, Maroc
}

doi: 10.19044/esj.2017.v13n7p124 URL:http://dx.doi.org/10.19044/esj.2017.v13n7p124

\begin{abstract}
This paper presents a partial equilibrium model of the credit market where the interference between nominal exchange rate, banks' balance sheet and credit channels of monetary policy transmission give rise to an incomplete pass through to the lending rate. The model shows that the overlapping of these three channels under floating exchange rate regime and external debt with endogenous risk premium is the cause of a conflictual effect of monetary shocks on banks behavior. This is in line with research highlighting externalities of floating exchange rate regime.
\end{abstract}

Keywords: Monetary Policy, Exchange rate, Credit Market, Partial Equilibrium Model

\section{Résumé}

Cet article présente un modèle d'équilibre partiel du marché du crédit où l'interférence entre le canal du taux de change, le canal du bilan des banques et le canal du crédit est à l'origine d'une distorsion nuisible à la complétude de la transmission de la politique monétaire au taux débiteur. Les résultats du modèle montrent que l'imbrication de ces trois canaux en régime de change flottant altère la répercussion des chocs monétaires sur le comportement des banques ayant une dette extérieure et supportant une prime de risque endogène. Ainsi, ce travail s'inscrit dans la perspective de mettre en lumière les externalités du régime de change flottant.

Mots clés : Politique monétaire, Taux de change, Marché du crédit, Modèle d'équilibre partiel

\section{Introduction}

La raison d'être de la banque centrale consiste à préserver la valeur réelle du bien dont elle détient le monopole de production, en l'occurrence la monnaie fiduciaire qui n'est autre qu'un lien social de confiance (Aglietta et 
Orléan, 2002). Pour ce faire, elle veille sur la stabilité des prix comme gage de la stabilité du pouvoir d'achat intrinsèque aux actifs monétaires. C'est ainsi que le ciblage d’inflation est prescrit aux petites économies ouvertes à régime de change flottant dans le cadre de leur politique monétaire. Du point de vue macroéconomique, la dite politique monétaire agit in fine sur le niveau des prix en agissant préalablement sur l'ensemble des composantes de la demande agrégée. Reste que, du point de vue opérationnel, sa mise en œuvre se fait via une batterie d'instruments permettant à la banque centrale d'intervenir sur le marché monétaire en vue d’influer sur ses valeurs d’équilibre, aussi bien en prix qu'en quantité. Ainsi, la répercussion des décisions de la banque centrale sur le niveau des prix nécessite un ensemble de canaux de transmission reliant le marché monétaire aux grandeurs macroéconomiques. Ces canaux vont du canal keynésien du taux d’intérêt au canal de l'incertitude et des signaux (Tang, 2015 ; Melosi , 2016), passant par le canal du taux de change et le canal du crédit (Bernanke et Blinder, 1988 ; Bernanke et Gertler, 1995), ainsi que le canal des actifs financiers et immobiliers (Mishkin, 2001; Mishkin, 2007) et le canal du capital des banques (Van Den Heuvel, 2002 ; Gambacorta et Shin, 2016). Si bien que l'essor de la politique monétaire non conventionnelle a mis en avant d'autres mécanismes de transmission qui viennent se superposer à ces canaux traditionnels.

Dans cet article, il ne s’agit guère de mettre en parallèle ces canaux de transmission, non moins de les juxtaposer, mais de mettre chacun de ces canaux en perspective de l'autre et d'élucider les liens d'endogénéité qu'ils entretiennent les uns avec les autres. Quand bien même que ce travail a pour objet uniquement la première phase du processus de transmission de la politique monétaire, à savoir la transmission des variations du taux directeur aux conditions bancaires, précisément le taux d’intérêt débiteur appliqué au marché du crédit bancaire. A première vue, le choix de cette perspective microéconomique renvoie à une approche sectorielle incapable de capter l'interférence entre les canaux de transmission. Mais à bien considérer les choses, ce choix permet de focaliser l'analyse sur un seul canal, à savoir le canal du crédit bancaire, et de démontrer que celui-ci implique d'autres canaux, en particulier le canal du bilan des banques et le canal du taux de change nominal. Ceci dit, l'idée de ce travail consiste à imbriquer le canal du taux de change dans le canal du bilan des banques, ce dernier étant lui-même imbriqué dans le canal du crédit bancaire. Il s’agit, au demeurant, de rejeter l'hypothèse de parallélisme entre les canaux et d'aller au-delà de la logique d'addition vers une logique d'imbrication, dans un modèle où s'entremêlent la transmission de la politique monétaire et les imperfections inhérentes au marché financier. A cet égard, faire appel aux imperfections financières est indispensable à cette imbrication et la principale imperfection dont il est 
question dans ce travail concerne la prime de financement externe que subissent les banques et qui est supposée être inversement liée à leur valeur patrimoniale. L'objectif est de démontrer que si le flottement du taux de change nominal fortifie la liaison entre les instruments maniés par banque centrale et la demande extérieure, son interférence avec le canal du bilan des banques et le canal du crédit peut être à l'origine d'une distorsion nuisible à la complétude de la transmission de la politique monétaire au taux d'intérêt débiteur. Il est question, en d'autres termes, de démontrer que ces trois canaux interagissent de sorte à interrompre la répercussion des décisions de la banque centrale sur le comportement des banques commerciales

Quel est le mécanisme sous-jacent à cette distorsion? Quelle est la nature de cette distorsion ? Pourquoi et quand est ce que cette distorsion s'enclenche ? Comment y pallier ou, du moins, comment limiter ses effets ? Autant de questions auxquelles tente de répondre cet article en étudiant le comportement optimal des banques commerciales dans le cadre d'un modèle d'équilibre partiel en se basant sur l'enseignement de la microéconomie bancaire (Freixas et Rochet, 2008). Ainsi, les banques sont appréhendées dans une optique d'organisation industrielle où le taux directeur devient le cout marginal de l'activité d’intermédiation bancaire. Les variations de ce cout sont répercutées sur le prix de vente des crédits qui n’est autre que le taux débiteur auquel sont octroyés les crédits. Dans ce modèle, la demande des crédits émane des entreprises, alors que l’offre est assurée par un oligopole de banques commerciales qui collectent les dépôts en vue de les transformer en prêts. Ces banques sont soumises à la réserve obligatoire et détiennent des bons du trésor dont le taux est assimilé au taux directeur de la banque centrale. Les normes prudentielles sont introduites dans le modèle et concernent exclusivement le capital réglementaire. A cet égard, les banques ont l'obligation de détenir des fonds propres et une dette obligataire subordonnée proportionnellement à leur portefeuille d'actifs pondérés par le risque. Les obligations émises par les banques le sont aussi bien à l'intérieur qu’à l'extérieur et la dette extérieure est libellée en monnaie étrangère et exprimée en monnaie nationale. Par ailleurs, le taux de change nominal est flexible et son flottement engendre un gain ou une perte de capital selon qu'il s’agisse d'une déprécation ou d'une appréciation. En outre, les variations du taux de change ont pour cause les variations du taux directeur selon la théorie de la parité non couverte du taux d’intérêt. Aucune restriction sur la mobilité des capitaux n’est prévue, sauf une imparfaite substituabilité entre la dette intérieure et la dette extérieure des banques. Dans cet ordre d'idées, la structure du modèle est présentée en première section et ses résultats ainsi que la discussion de ses implications économiques font l'objet de la deuxième section. L'article conclut sur les limites auxquelles se heurtent le modèle et les perspectives de recherche qu'il offre. 


\section{Modèle d'équilibre partiel}

L'offre du crédit $L$ est du ressort des banques commerciales qui, pour ce faire, collectent les dépôts à terme des résidents $D_{h}$ et ceux des nonrésidents $D_{e}$, qu'elles rémunèrent indistinctement au même taux créditeur $i_{D}$. La gestion de la trésorerie et la prévention des chocs de liquidité impliquent la détention de réserves bancaires dont une fraction est confiée à la banque centrale dans le cadre de la réserve obligatoire $R O$, le reste étant détenu en principe sous forme d'encaisses oisives ou idéalement sous forme d'actifs relativement liquides et peu risqués, en l'occurrence les obligations d'Etat $B T$.

Ainsi :

$$
R O=r \cdot D
$$

Avec $r$ le ratio des réserves obligataires fixé par la banque centrale et :

$$
D=D_{h}+D_{e}
$$

Par ailleurs, les banques sont astreintes à observer des ressources stables par rapport à leurs emplois pondérés par le risque. Ces ressources stables sont constituées des fonds propres, supposées être prédéterminées à $\bar{E}$ ainsi que des titres obligataires $B_{h}$ émis sur le marché financier national et des titres obligataires $B_{e}$ émis sur le marché international. Les obligations $B_{e}$ sont libellées en devises et multipliées par le taux de change nominal à l'incertain $Z$ pour être exprimées en monnaie nationale. Ceci étant, le dit ratio de prudence relatif au capital réglementaire peut être formulé comme suit :

$$
\bar{E}+B_{h}+Z \cdot B_{e}=\rho \cdot \sigma \cdot L
$$

Le coefficient $\rho$ est déterminé selon les normes prudentielles en vigueur et $\sigma$ est le coefficient de pondération du risque inhérent aux crédits bancaires que les banques estiment selon leurs méthodes d'évaluation internes. Chacune des composantes des ressources stables occupent un poids donné et la dissociation des fonds propres par rapport aux titres obligataires aboutit à l'écriture suivante :

$$
\left\{\begin{aligned}
\bar{E} & =(1-\gamma) \cdot \rho \cdot \sigma \cdot L \\
B_{h}+Z \cdot B_{e} & =\quad \gamma \cdot \rho \cdot \sigma \cdot L
\end{aligned}\right.
$$

Avec $\gamma$ le poids des actions et $1-\gamma$ le poids des obligations. La distinction entre les obligations nationales et internationale donne l'écriture suivante :

$$
\left\{\begin{aligned}
B_{h} & =\quad \beta \cdot \gamma \cdot \rho \cdot \sigma \cdot L \\
Z \cdot B_{e} & =(1-\beta) \cdot \gamma \cdot \rho \cdot \sigma \cdot L
\end{aligned}\right.
$$

Avec $\beta$ le poids de la dette obligataire domestique et $1-\beta$ le poids de la dette obligataire internationale.

Quant à la contrainte budgétaire des banques, elle surgit du bilan agrégé suivant : 


$$
L+B T+R O=\bar{E}+B_{h}+Z \cdot B_{e}+D_{h}+D_{e}
$$

Afin de déterminer le montant des bons du trésor de façon résiduelle, la contrainte budgétaire en équation (6) est réécrite comme suit :

$$
B T=\bar{E}+B_{h}+Z \cdot B_{e}+D_{h}+D_{e}-L-R B
$$

Quant à l'actif net $A N_{b}$ des banques, il est :

$$
A N_{b}=L+B T+R B-B_{h}-Z \cdot B_{e}-D_{h}-D_{e}
$$

Sachant que :

$$
Z \cdot B_{e}=Z_{0} \cdot B_{e}+\Delta Z \cdot B_{e}
$$

Avec, $Z_{0}$ le taux de change nominal initial et $\Delta Z$ la variation du taux de change.

L'actif net peut donc être réécrit comme suit :

$$
\begin{aligned}
& A N_{b}=\left(L+B T+R B-B_{h}-Z_{0} \cdot B_{e}-D_{h}\right. \\
& \left.\quad-D_{e}\right)-\Delta Z \cdot B_{e}
\end{aligned}
$$

Etant donné que le montant des capitaux propres est prédéterminé $\bar{E}$, l'actif net des banques commerciales dépend d'une seule variable, à savoir la variation du taux de change nominal $\Delta Z$. En effet, une appréciation (dépréciation) du taux de change nominal à l'incertain représente un renchérissement (réduction) de la dette extérieure libellée en devise et exprimée en monnaie nationale. Cela signifie que l'appréciation (dépréciation) de la valeur de la monnaie étrangère par rapport à la monnaie nationale augmente (baisse) le montant en monnaie nationale de la dette extérieure contractée en monnaie étrangère. Il s'agit donc de pertes (gains) de change que les banques subissent. Défini comme étant la différence entre la sommes des actifs et la sommes des dettes, le patrimoine des banques s'en trouvé forcément touché, d’où la relation suivante :

$$
A N_{b}=f(\Delta Z)
$$

Avec $f^{\prime}<0$.

Par ailleurs, le taux obligataire $i_{B}$ auquel les banques empruntent sur le marché financier domestique est déterminé par référence au taux $i_{R}$ des bons du trésor de la même maturité. Ce taux de référence représente le taux sans risque et il est assimilé au taux directeur auquel se refinancent les banques commerciales auprès de la banque centrale. Ce taux est donc augmenté d'une prime de risque $\theta^{b}$ afin d'obtenir le taux que supportent les banques sur le marché obligataire :

$$
i_{B}=i_{R}+\Theta
$$


Cette équation reflète la structure par risque du taux obligataire payé par les banques commerciales. La prime de risque $\Theta$ est supposée être endogène et varie en fonction du risque perçu par les prêteurs et encouru par les banques. Plus ces dernières sont de grande taille et disposent d'un portefeuille d'actifs sain, plus elles sont perçues comme des entreprises relativement moins risquées. De même, plus leurs exigibilités sont encombrantes, plus le marché les jugent comme relativement risquées et leur afflige une prime de financement externe supplétive. Ainsi, une relation inverse est établie entre l'actif net des banques et la prime de risque qu'elles subissent :

Avec $g^{\prime}<0$.

$$
\Theta=g\left(A N_{b}\right)
$$

Compte tenu de l'équation (11) qui capte la relation entre l'actif net des banques et le taux de change nominal, l'équation (13) peut être réécrite comme suit :

$$
\Theta=g \circ f(\Delta Z)
$$

Ainsi, la prime de risque supportée par les banques commerciales dépend des fluctuations du taux de change nominal. Intuitivement, l'appréciation du taux de change à l'incertain constitue une dégradation de la valeur patrimoniale des banques et une détérioration de leur solvabilité qui se répercute sur la prime de risque qu'elles supportent au marché obligataire. Au contraire, une dépréciation du taux de change à l'incertain améliore la qualité perçue du bilan des banques commerciales et réduit la prime de risque qu'elles subissent.

S'agissant des fluctuations à court terme du taux e change nominal, celles-ci sont le fruit d'un comportement d'arbitrage de la part des épargnants, aussi bien les résidents que les non-résidents, qui ajustent leur portefeuille d'actifs en fonction du couple risque et rendement qui leur est associé. Ce faisant, ils égalisent le taux de référence domestique $i_{R}$ avec son cout d'opportunité qui est le taux de référence à l'étranger $i_{R}^{*}$, compte tenu du taux de change auquel ils substitueraient actuellement leur placement et celui auquel ils anticipent de récupérer leur rendement. De là, la parité non couverte des taux d'intérêt est :

$$
i_{R}=\frac{Z^{a}}{Z} \cdot i_{R}^{*}
$$

Avec $Z^{a}$ le taux de change nominal à l'incertain anticipé. Eu égard la nature statique de ce modèle, $Z^{a}$ est supposé être exogène et fixé à une valeur constante $\overline{Z^{a}}$. Même, le taux de référence à l'étranger $i_{R}^{*}$, qui n'est autre que le taux des bons du trésor émis par un gouvernement étranger servant de référence au vue de sa solvabilité, sa valeur est prédéterminée à l'extérieur de l'économie et considérée comme variable exogène. 
Sachant ceci, l'équation (15) peut être reformulée ainsi :

$$
Z=\frac{i_{R}^{*}}{i_{R}} \cdot \overline{Z^{a}}
$$

Compte tenu de l'égalité suivante :

$$
Z_{0}+\Delta Z=Z
$$

Les fluctuations du taux de change s'expriment donc par :

$$
\Delta Z=\frac{i_{R}^{*}}{i_{R}} \cdot \overline{Z^{a}}-\overline{Z_{0}}
$$

Où $i_{R}$ est la seule variable à pouvoir influer sur le sens et l'amplitude des fluctuations du taux de change. Ce dernier est donc une fonction négative du taux directeur fixé par la banque centrale :

Avec $h^{\prime}<0$.

$$
\Delta Z=h\left(i_{R}\right)
$$

En combinant les équations (14) et (20), la prime de risque peut donc être exprimée en fonction du taux directeur :

En posant :

$$
\Theta=g \circ f \circ h\left(i_{R}\right)
$$

$$
g \circ f \circ h\left(i_{R}\right)=\theta\left(i_{R}\right)
$$

Le paramètre $\Theta$ qui capte la prime de risque devient une fonction $\theta$ du taux directeur ayant la forme réduite suivante :

$$
\Theta=\theta\left(i_{R}\right)
$$

Avec $\theta^{\prime}<0$. Ceci mène à réécrire le taux obligataire supporté par les banques, tant sur leur dette intérieure qu'extérieure, comme suit :

$$
\left\{\begin{array}{l}
i_{B}=i_{R}+\theta\left(i_{R}\right) \\
i_{B}^{*}=i_{R}^{*}+\theta\left(i_{R}\right)
\end{array}\right.
$$


Avec $i_{B}^{*}$ le taux obligatoire supporté sur la dette extérieure et qui est égal au taux d'intérêt de référence à l'étranger $i_{R}^{*}$ augmenté de la prime de risque endogène. De par son effet sur le taux de change nominal, toute modification du taux directeur affecte la valeur patrimoniale des banques et agit indirectement sur la prime de risque qu'elles supportent. Ainsi, les changements opérés sur le taux directeur et qui s’inscrivent dans le cadre opérationnel de la politique monétaire, sont doublement transmis au taux obligataire $i_{B}$ facturé sur la dette intérieure des banques. En effet, en tant que valeur de référence du taux obligataire, le taux directeur l'influence directement et au même sens, mais en tant qu'élément déterminant de sa structure par risque, il le modifie indirectement et en sens inverse. Cet effet conflictuel qu'exerce le taux directeur sur le taux obligataire de la dette intérieur, disparait quand il s'agit du taux obligataire de la dette extérieure $i_{B}^{*}$ qui ne réagit qu'indirectement, à travers la prime de risque, et en sens inverse à toute modification du taux de refinancement.

Ceci dit, le profit des banques $\pi_{b}$ est donc :

$$
\begin{array}{r}
\pi_{b}=\left(1+i_{L}\right) \cdot p \cdot L+\left(1+i_{R}\right) \cdot B-\left(1+i_{D}\right) \cdot D \\
-\left(1+i_{B}\right) \cdot B_{i}-\left(1+i_{B}^{*}\right) \cdot Z \cdot B_{e}^{*}
\end{array}
$$

Avec $p$ et $i_{D}$ respectivement la probabilité de remboursement des crédits consentis et le taux créditeur auquel sont collectés les dépôts et qui sont, tous les deux, supposés être exogènes. A noter que le taux créditeur est déterminé par la confrontation de l'offre et la demande sur le marché des dépôts, selon un mécanisme qui est échappe à la portée de cette analyse. En l'absence des couts fixes et variables afférents à l'activité d'intermédiation financière et en faisant abstraction des autres sources de gain, le profit des banques est une marge d'intérêt entre, d'un côté, le taux débiteur sur les crédits probablement remboursés $p \cdot L$ en plus du rendement des bons du trésor et, de l'autre côté, le taux créditeur payés sur les dépôts ainsi que les taux des dettes obligataires. A noter que, eu égard à la nature statique et unipériodique de ce modèle, les banques payent et touchent à la fois les intérêts et la capital.

Le programme d'optimisation des banques consiste donc à maximiser le profit $\pi_{b}$ en équation (25), sous la contrainte budgétaire en équation (6) où la dette obligataire est remplacée par son expression en équation (5).

Ce programme doit être résolu compte tenu de la fonction de demande des crédits adressée aux banques :

$$
L=l\left(i_{L}\right)
$$

Avec $l^{\prime}<0$. 


\section{Résultats et discussion}

La condition de premier ordre du programme d'optimisation (Section 1) des banques commerciales permet d'obtenir la formule du taux débiteur optimal $\left(1+i_{L}\right)$ :

$$
\frac{\left(1+i_{R}\right) \cdot(1-\gamma \cdot \rho \cdot \sigma)+\left(1+i_{B}\right) \cdot \beta \cdot \gamma \cdot \rho \cdot \sigma+\left(1+i_{B}^{*}\right) \cdot(1-\beta) \cdot(27}{p \cdot\left(1+\frac{1}{\varepsilon_{L}}\right)}
$$

Avec $\varepsilon_{L}$ l'élasticité de la demande des crédits bancaires dont l'expression est la suivante :

$$
\varepsilon_{L}=\frac{l^{\prime} / l}{i_{L}^{\prime} / i_{L}}
$$

En posant :

$$
\vartheta_{L}=p \cdot\left(1+\frac{1}{\varepsilon_{L}}\right)
$$

Le taux débiteur $\left(1+i_{L}\right)$ peut être réécrit comme suit :

$$
\begin{aligned}
\vartheta_{L} \cdot\left(1+i_{R}\right) \cdot(1-\gamma \cdot \rho \cdot \sigma)+\left(1+i_{B}\right) \cdot \beta \cdot \gamma \cdot \rho \cdot \sigma+\left(1+i_{B}^{*}\right) \\
\cdot(1-\beta) \cdot \gamma \cdot \rho \cdot \sigma
\end{aligned}
$$

Il s'agit d'une moyenne pondérée de trois variables, le taux directeur, le taux obligataire de la dette intérieure et le taux obligataire de la dette extérieure. Sachant que :

$$
(1-\gamma \cdot \rho \cdot \sigma)+\beta \cdot \gamma \cdot \rho \cdot \sigma+(1-\beta) \cdot \gamma \cdot \rho \cdot \sigma=1
$$

Cette moyenne pondérée constitue une sorte de «cout marginal moyen », à laquelle s'ajoute un " Mark up » $\vartheta_{L}$ qui, dans ce cas, représente la prime de risque exigée par les banques commerciales sur les crédits qu'elles distribuent.

En remplaçant les taux obligataires par leurs formules en équation (24), la formule du taux débiteur optimal peut être explicitée comme suit :

$$
\begin{aligned}
& \quad \begin{array}{c}
\left(1+i_{L}\right)=\vartheta_{L} \cdot\left(1+i_{R}\right) \cdot(1-\gamma \cdot \rho \cdot \sigma)+\left(1+i_{R}+\theta\left(i_{R}\right)\right) \\
\cdot(\beta \cdot \gamma \cdot \rho \cdot \sigma) \\
+\left(1+i_{R}^{*}+\theta\left(i_{R}\right)\right) \cdot((1-\beta) \cdot \gamma \cdot \rho \cdot \sigma)
\end{array}
\end{aligned}
$$


Le taux débiteur se trouve ainsi lié, à la fois directement et indirectement, au taux de refinancement auprès de la banque centrale. Le taux débiteur est dès lors le prix auquel les banques vendent les crédits bancaires et sur lequel se répercute le cout marginal. Ce dernier est manifestement dominé par le taux directeur, même lorsqu'elle s'agit du cout de la dette extérieure où le taux directeur apparait indirectement via la prime de risque. Néanmoins, l'effet net du taux directeur sur la composition du cout marginal des banques est à éclaircir. C’est pourquoi il faut déterminer le sens et l'ampleur de la transmission de la politique monétaire au taux débiteur en cherchant le signe et l'amplitude de la variation de ce dernier suite à une variation du taux directeur. Pour obtenir ce signe et cette amplitude, il suffit d'un simple exercice de dérivée partielle de la fonction du taux débiteur par rapport au taux directeur :

$$
\begin{gathered}
\frac{\partial i_{L}}{\partial i_{R}}=\vartheta_{L} \cdot\left[(1-\gamma \cdot \rho \cdot \sigma)+\left(1+\theta^{\prime}\right) \cdot(\beta \cdot \gamma \cdot \rho \cdot \sigma)+\theta^{\prime} \cdot(1-\beta) \cdot \gamma \cdot \rho\right. \\
\cdot \sigma]
\end{gathered}
$$

Sachant que $\theta^{\prime}<0$.

Par une légère manipulation algébrique, l'équation ci-dessus peut être reformulée comme suit :

$$
\frac{\partial i_{L}}{\partial i_{R}}=\vartheta_{L} \cdot\left[1+\gamma \cdot \rho \cdot \sigma \cdot\left(\theta_{i_{B T}}^{\prime}-1\right) \cdot(1-\beta)+\gamma \cdot \rho \cdot \sigma \cdot \theta_{i_{B T}}^{\prime} \cdot \beta\right]
$$

Dans ce cadre analytique, une expansion monétaire est mise en œuvre par la baisse implicite du taux de refinancement auprès de la banque centrale et devient explicitement établie dans le modèle à travers la baisse du taux des bons du trésor. Il s'agit là d'un cadre opérationnel de la politique monétaire où son instrument de taux d'intérêt, en l'occurrence le taux directeur, est étroitement lié à son objectif opérationnel, ici le taux obligataire de l'Etat, sachant que son objectif stratégique est essentiellement la stabilité des prix et accessoirement la relance de l'activité économique. Du côté des banques commerciales, la baisse du taux des bons du trésor constitue une baisse du cout d'opportunité de leur activité d'intermédiation financière, une baisse dont elles tiennent compte dans leur comportement arbitrage entre les maturités et les risques de leur portefeuille d'actifs. Par effet direct, l'octroi des prêts au secteur privé, plus risqué certes que la détention des titres publics et moins liquides, devient possible par une baisse du taux débiteur qui leur est associé. 
Par ailleurs, la baisse (hausse) du taux d'intérêt domestique relativement aux taux d'intérêt en vigueur à l'étranger entraine une sortie (entrée) de capitaux qui se traduit par une appréciation (dépréciation) du taux de change nominal à l'incertain, due essentiellement à l'achat massif de la monnaie étrangère (nationale) sur le marché des changes. A défaut d'instruments de couverture contre les risques inhérents à ses fluctuations non anticipés, le taux de change nominal se trouve au centre d'une relation d'arbitrage entre les actifs domestiques libellés en monnaie nationale et les actifs émis à l'étranger et libellés en monnaie étrangère. Cette relation n'est autre que celle qualifiée par la littérature économique de parité non couverte des taux d'intérêt. En effet, la baisse du taux de l'argent au jour le jour, lorsqu'elle se propage tout au long de la courbe des taux d'intérêt, rend moins attractif l'achat des actifs financiers nouvellement émis et représentatifs d'un droit créance. Les épargnants qui désertent le marché primaire se tournent conséquemment vers le marché secondaire et peuvent hypothétiquement, en l'absence de couts de transaction sur la mobilité des capitaux, aller vers le marché financier international. Dans le premier scénario, la valeur des titres négociables s’apprécie, alors que c’est la valeur de la monnaie étrangère exprimée en monnaie nationale qui s’apprécie dans le deuxième cas. En tout cas, la politique monétaire crée un cout d’opportunité pour les spéculateurs qui mettent en mouvement, à travers leurs opérations d'arbitrage, une appréciation de la valeur de marché des actifs financiers, en l'occurrence, le taux de change nominal à l'incertain. C'est ainsi que ce dernier acquiert la qualité de canal de transmission des chocs monétaires vers la sphère réelle de l’économie. Cependant, les banques doivent s’alimenter en liquidité en émettant des titres non soumis à la réserve obligatoire, en l'occurrence des titres de créances négociables tels que les certificats de dépôt ou carrément des obligations à diverses maturités selon le besoin. Dans ce cas, les banques font face, à l'instar des entreprises, à une prime de financement externe qui est une prime de rossignol à la Akerlof (1970). Il s'agit surtout d'une prime de risque eu égard à l'asymétrie d'information qui subsiste sur la qualité de leurs actifs qui serviront de collatéral en cas de défaut. Une discipline de marché est à observer dès lors et toute banque peu ou relativement moins capitalisée serait perçue comme très ou relativement plus risquée par rapport aux autres, la prime de risque qu'elle supporte est donc inversement liée à la valeur de son patrimoine. Etant donné qu'une partie de la dette des banques est libellée en devises et eu égard à l'appréciation du taux de change nominal, les banques assistent à une détérioration de leurs bilans, une détérioration due essentiellement à une diminution de leur valeur patrimoniale concrétisée par une hausse de la valeur en monnaie nationale de leur dette et une baisse conséquente de la valeur de marché de leur actif net. Cette dégradation du patrimoine des 
banques, dans sa dimension de collatéral à l'endettement, aussi bien interne qu'externe, signifie une hausse du risque perçu par le marché financier et, par voie de conséquence, une hausse de la prime de risque exigée sur les prêts qui leurs sont accordés. Cette hausse de la prime de risque apparait sur le taux obligataire supporté sur la dette intérieure et extérieure. Le taux obligataire sur la dette intérieure étant le taux des bons du trésor augmenté d'une prime de risque et le taux obligataire sur la dette extérieure étant le taux international sans risque augmenté de la dite prime. Eu égard à ces mécanismes en chaine, la baisse du taux directeur se traduit, in fine, par une hausse du taux obligataire sur la dette intérieure et extérieure. De ce fait, une politique monétaire expansionniste produit à un " effet conflictuel » sur le taux débiteur. D'un côté, elle réduit sa valeur par un effet de cout d'opportunité direct, de l'autre côté, elle augmente sa valeur par un effet de cout marginal indirect. L'effet net dépend essentiellement de l'élasticité de la prime de risque aux variations du taux de change et de l'élasticité de ce dernier au taux directeur. En somme, l'effet du taux de change sur la prime de risque réduit la complétude de la transmission de la politique monétaire. En outre, cette incomplétude de la transmission de la politique monétaire dépend de la part de la dette extérieure dans le passif des banques. Plus celleci est importante, plus cette incomplétude est intense. Plus les banques s'endettent à l'extérieur, plus la transmission de la politique monétaire est incomplète. C'est ce que montrent clairement les cas extrêmes suivants :

Sachant que :

$$
\frac{\partial i_{L}}{\partial i_{R}}= \begin{cases}\vartheta_{L} \cdot\left(1+\gamma \cdot \rho \cdot \sigma \cdot\left(\theta_{i_{R}}^{\prime}-1\right)\right), & \beta=0 \\ \vartheta_{L} \cdot\left(1+\gamma \cdot \rho \cdot \sigma \cdot \theta_{i_{R}}^{\prime}\right) & \beta=1\end{cases}
$$

$$
\left|\gamma \cdot \rho \cdot \sigma \cdot\left(\theta_{i_{B T}}^{\prime}-1\right)\right|>\left|\gamma \cdot \rho \cdot \sigma \cdot \theta_{i_{B T}}^{\prime}\right|
$$

Ainsi, en régime de change flottant, la transmission de la politique monétaire au taux débiteur est incomplète lorsque les banques supportent une dette extérieure conjuguée à une prime de risque endogène. Cette incomplétude provient de l'effet du taux de change sur l'actif net des banques et s'accentue avec le poids relatif de la dette extérieure.

En termes d'élaboration et de mise en œuvre de la politique monétaire, ces résultats indiquent que la complétude de la transmission aux conditions bancaires est altérée par l'effet conflictuel du taux directeur sur le taux débiteur et qu'elle est rétablie par la prise en compte du lien, certes implicite, entre le flottement du taux de change nominal et la prime de risque supportée par les banques sur leur dette obligataire. 


\section{Conclusion}

Cet article met en lumière l'interférence entre trois principaux canaux de transmission de la politique monétaire, à savoir le canal du taux du crédit, le canal du bilan des banques et le canal du taux de change nominal. Compte tenu de la liaison entre la valeur patrimoniale des banques et la prime de risque sur la dette obligataire en tant qu'imperfection financière, cette interférence s'avère être à l'origine d'une distorsion nuisible à la complétude de la transmission de la politique monétaire. Les banques, ayant une dette extérieure libellée en devise et exprimée en monnaie nationale, supportent une prime de risque inversement liée à leur valeur patrimoniale. Dans ces conditions, les fluctuations du taux de change nominal influent négativement sur le taux obligataire payé par les banques et, en conséquent, la politique monétaire agit directement sur le taux directeur et indirectement et au sens opposé sur le taux obligataire, les deux étant les principales composantes du cout marginal des banques. En effet, en tant que valeur de référence du taux obligataire, le taux directeur l'influence directement et au même sens, mais en tant qu'élément déterminant de sa structure par risque, il le modifie indirectement et en sens inverse.

Il n'en demeure pas moins que cet article se heurte à ses propres limites et que des voies d'amélioration et d'extension sont potentiellement envisageables.

Dans ce modèle, la structure par risque du taux débiteur présuppose que celui-ci est défini par rapport au taux obligataire, tant public que privé, augmenté d'un spread exogène qui reflète la prime de risque associée à la probabilité de remboursement des prêts consentis au secteur privé. Or, cette exogénéité de la prime de risque est une simplification aberrante de la complexité extrême qui sous-tend la détermination du taux débiteur. Il s’agit donc d'une hypothèse restrictive et peu réaliste qui doit être relâchée afin de tenir compte, entre autres, de l'effet du cycle économique sur la perception du risque par les banques.

Pour ce faire, la prime de risque doit être endogène et devrait dépendre de la valeur de marché du collatéral mis en jeu dans les opérations de crédit, ce collatéral étant le patrimoine des emprunteurs. Ce faisant, la prime de risque serait pro-cyclique car, en étant inversement liée à la valeur de l'actif net des entreprises et ménages, elle accentue les phases des cycles économiques et financiers. Toutefois, une telle extension n’est faisable qu'à travers une combinaison de l’équilibre du marché des crédits avec l'équilibre du marché réel dans un modèle d’équilibre général. 
Ce passage d'une analyse purement microéconomique vers une approche macroéconomique, outre l'étendue de l'analyse qu'il offre, permettrait également d'identifier une deuxième distorsion de la transmission des chocs monétaires au taux débiteur et dont la cause demeure la flexibilité du taux de change nominal. En supposant que parmi les éléments du bilan des emprunteurs figurent des postes libellés de devises, les fluctuations du taux de change constitueraient dès lors une source de variation de la valeur de leur patrimoine. Le sens et l'amplitude de cette variation est de nature à influer négativement sur la prime de risque qu'ils subissent lorsqu'ils contractant des crédits et une seconde distorsion vient se superposer à celle déjà établie dans cet article. Le taux de change nominal figurerait doublement sur la formule du taux débiteur optimal, premièrement au niveau de la prime de risque supportée par les banques commerciales et deuxièmement au niveau de la prime de risque supportée par les emprunteurs auprès des dites banques commerciales. Tout compte fait, formaliser cette double distorsion est vraisemblablement une piste à explorer et qui fait l'objet d'un travail de recherche en cours.

Par ailleurs, la parité non couverte des taux d'intérêt constitue l'un des soubassements théoriques de la distorsion soulevée par cet article. Or, le mécanisme d'arbitrage sous-entendu par cette parité n’est pas aussi systématique qu'il semble l'être et demeure sujet à caution eu égard à la validité empirique dont il manque. Ainsi, il convient de restreindre la mobilité des capitaux et la substituabilité des actifs étrangers aux actifs domestiques. Cette restriction, selon la sévérité désirée, peut aller de simples couts de transaction et d'ajustement de portefeuille vers un control règlementaire pur et dur, passant par des mécanismes de régulation tels que la taxation. En l'introduisant dans le modèle, les flux de capitaux suivant un choc monétaire baissent, les fluctuations du taux de change nominal qui l'accompagnent diminuent et la distorsion soulevée dans cet article s'en trouve ainsi allégée. Néanmoins, cette rectification doit obligatoirement être faite dans le cadre d'un modèle macroéconomique afin de tenir compte de ses effets d’équilibre général. Car si cette restriction rend plus réactif le taux débiteur aux chocs de la politique monétaire et fortifie la transmission de ses effets via le canal du crédit, cela se fait sans doute au détriment de la bonne transmission des décisions de la banque centrale vers la demande extérieure via le canal du taux de change. 
Dans ce modèle, les banques sont supposées avoir un élément du passif libellé en devise alors qu'aucun de leurs actifs ne l'est. C'est pourquoi les fluctuations du taux de change agissent en sens inverse de l'effet du taux directeur. A titre d'illustration, les résultats du modèle montrent qu'une hausse de ce dernier attire les capitaux et déprécie le taux de change nominal à l'incertain, faisant baisser la valeur en monnaie nationale de la dette extérieure des banques et réduisant ainsi la prime de risque qu'elles subissent. Ce faisant, alors que le cout marginal augmente par la hausse du taux de refinancement auprès de la banque centrale, il baisse par la baisse de la prime de risque sur la dette obligataire. Cela est dû essentiellement à la structure du bilan qui présuppose la construction de ce modèle et qu'il suffit de modifier en supposant que les banques détiennent des actifs libellés en devises, indirectement via leurs filiales installées à l'étranger ou directement à travers des crédits domestiques consentis en devises. Toutefois, la conséquence d'une telle modification sur les résultats de ce modèle dépend largement de la proportion des actifs en devises par rapport à la dette extérieure des banques et du gain de change qui en résulte. Cela varie selon qu'il s'agisse de paramétrer la modélisation en vue d'obtenir le résultat escompté ou de reproduire une réalité factuelle à travers des équations sans préalable théorique.

\section{References:}

1. Aglietta, M., \& Orléan, A. (2002). La monnaie entre violence et confiance. Paris: Odile Jacob.

2. Akerlof G. A. (1970). The Market for "Lemons": Quality Uncertainty and the Market Mechanism. The Quarterly Journal of Economics, 84(3), 488-500.

3. Bernanke, B. S., \& Blinder, A. S. (1988). Credit, money, and aggregate demand. American Economic Review, 78, 435-439.

4. Bernanke, B. S., \& Gertler, M. (1995). Inside the black box: the credit channel of monetary policy transmission. Journal of Economic Perspectives, 9, 27-48.

5. Freixas, X., \& Rochet J.C. (2008). Microeconomics of Banking. Cambridge, MA: MIT Press.

6. Gambacorta L. \& Shin H. S. (2016). Why bank capital matters for monetary policy (BIS Monetary and Economic Department Working Paper No. 558). Basel: Bank for International Settlements.

7. Melosi, L. (2016). Signaling Effects of Monetary Policy (FRBC Working Paper, No. 2016-14). Chicago: Federal Reserve Bank of Chicago. 
8. Mishkin, F. S. (2001). The Transmission Mechanism and the Role of Asset Prices in Monetary Policy (NBER Working Papers No. 8617), Cambridge: National Bureau of Economic Research.

9. Mishkin, F. S. (2007). Housing and the Monetary Transmission Mechanism (Finance and Economics Discussion Series 2007-40). Washington: Board of Governors of the Federal Reserve System.

10. Tang, J. (2015). Uncertainty and the Signaling Channel of Monetary Policy (FRBB Working Paper No. 15-8). Boston: Federal Reserve Bank of Boston.

11. Van Den Heuvel, S. (2002). Does bank capital matter for monetary transmission. Economic Policy Review, Issue May, 259-265. 